\title{
CONSUMO DE ALCOHOL ENTRE ESTUDIANTES DE MEDICINA QUE CURSAN ASIGNATURAS DE FARMACOLOGIA I Y II.
}

\section{Alcohol consumption among students of pharmacology I and II.}

\author{
*Vilma Gómez, *Mario Reyes, *Pablo Galo, *Jeancarlo Euceda, *Lissandro Agurcia, *Iveth Licona \\ ** Lourdes Azucena Andrade Avila.
}

\section{RESUMEN}

Antecedentes: El alcohol es considerada una droga que tiene repercusión negativa en la vida del individuo consumidor activo o no. El consumo de alcohol es multifactorial y preocupa en jóvenes universitarios por ser una droga de fácil acceso, barato y con menos estigma a nivel social. Objetivo: Describir y comparar el consumo de alcohol en estudiantes de medicina hombres y mujeres que cursan las asignaturas de Farmacología | y || el primer semestre del año 2017 en la Escuela Universitaria de Ciencias de la Salud en la Universidad Nacional Autonoma de Honduras en el Valle de Sula (EUCS/ UNAH-VS). Pacientes y métodos: Estudio cuantitativo, alcance descriptivo. Población: muestra por conveniencia de 56 estudiantes (28 mujeres y 28 hombres). Se utilizó un instrumento tipo cuestionario para la recolección de datos, previo consentimiento informado. Resultados: El 73\%. (41) consumía alcohol. Los resultados revelaron mayor consumo en mujeres $43 \%$ (24) que en hombres $30 \%$ (17). Un $36.7 \%$ (20) refiere la ingesta de alcohol en vacaciones y $26.8 \%$ (15) después de exámenes. Un $98 \%$ (55) conocían de los efectos nocivos del alcohol. Conclusiones: El consumo de alcohol es una práctica en los jóvenes universitarios de las asignaturas de Farmacología I y II. Un alto porcentaje de los estudiantes partícipes de éste estudio ha consumido alcohol, la mayoría lo hace ocasionalmente. El consumo es mayor por parte de las mujeres. La sustancia más consumida es la cerveza (ambos sexos). La mayoría

\footnotetext{
*Estudiantes de quinto año de la Carrera de Medicina de la Escuela Universitaria Ciencias de la salud de la Universidad Nacional Autónoma de Honduras en el Valle de Sula (EUCS/UNAH-VS).

** Especialista en Anestesiología y docente EUCS/UNAH-VS

Dirigir correspondencia a vilmaalegomez@gmail.com

Recibido: 15 de octubre del 2017 Aprobado: 1 de mayo del 2018
}

conoce los efectos nocivos del alcohol, pero no los detiene a consumirlo.

\section{PALABRAS CLAVE}

Alcohol, estudiante universitario, sustancias nocivas.

\begin{abstract}
Background: Alcohol is considered a drug that has a negative impact on the life of the individual, whether they are consumers or not. The consumption of alcohol is multifactorial and worries university students because it is an easily accessible, cheap drug with less social stigma. Objective: To describe and compare the consumption of alcohol in medical students men and woman who attend the subjects of pharmacology I and II in the first semester of 2017 in the Escuela Universitaria de Ciencias de la Salud de la Universidad Nacional Autónoma de Honduras en el Valle de Sula, EUCS/UNAH-VS. Patients and Methods: A quantitative study with descriptive scope. Population by convenience, of 56 students ( 28 women and 28 men) who attended pharmacology class I and II. A questionnaire type instrument was used to collect data with prior informed consend. Results: $73 \%$. (41) consumed alcohol. The results revealed higher consumption in women $43 \%$ (24) than in men $30 \%$ (17). A $36.7 \%$ (20) refers to the alcohol intake during vacations and $26.8 \%$ (15) after exams. $98 \%$ (55) were aware of the harmful effects of alcohol. Conclusions: Alcohol consumption is a practice among university students in the Pharmacology in the subjects I and II. The majority of students who participated in the in this study have consumed alcohol, and a percentaje do so occasionally. Consumption is higher on women. The most consumed substance is beer (both sexes).
\end{abstract}


Most know the harmful effects of alcohol, but it does not stop them from consuming it.

\section{KEYWORDS}

Ethanol, noxae, students medical.

\section{INTRODUCCIÓN}

El consumo de alcohol en la población en general es un problema representativo para la salud de cada individuo. La problemática derivada del consumo de alcohol en los jóvenes es diferente a la del adulto. En los jóvenes, los efectos negativos suelen referirse a alteraciones de las relaciones con la familia, compañeros y maestros, bajo rendimiento escolar, agresiones, violencias, perturbación del orden público y conductas de alto riesgo. ${ }^{(1)}$ Conducir tras haber bebido, tener actividades sexuales sin protección que conllevan embarazos no deseados y enfermedades de transmisión sexual son algunas de estas conductas. En general, los estudiantes que beben grandes cantidades de alcohol, comparados con los que no beben, exhiben más conductas de riesgo para sí mismos y para los demás. ${ }^{(1)}$

Existen grupos específicos de riesgo para el consumo de alcohol; pero sin importar la razón, la consecuencia del consumo de alcohol siempre es la misma; repercute en la salud física y mental del individuo, orientándolo a conductas de riesgo. ${ }^{(2)}$ En la actualidad el fácil acceso a esta sustancia psicoactiva se ha vuelto un factor importante para que jóvenes se presten a su utilización y aumenten la posibilidad de causar dependencia y alteraciones psicológicas a tempranas edades.

El término alcoholismo se refiere al consumo crónico y continuado o al consumo periódico de alcohol que provoca un deterioro del control sobre la bebida, episodios frecuentes de intoxicación y obsesión por el alcohol. ${ }^{(3)}$ La utilización de etanol sin importar su fin recreativo significa totalmente un riesgo a la salud, ya que se hace referencia al consumo de alcohol como una conducta de alto riesgo que se desarrolla a edades cada vez más tempranas. ${ }^{(4)}$ También se define al alcohol como la sustancia legal de consumo más común en el mundo. ${ }^{(5)}$ Una buena parte de la población universitaria acepta haber tenido contacto al menos una vez con etanol, y muchos de estos después de los 16 años. ${ }^{(6)}$

Personas que a temprana edad no fueron consumidores activos podrían crear un hábito de consumo como para combatir el estrés. Una gran parte de los estudiantes universitarios presentan niveles de estrés de moderado a alto, volviéndose vulnerables al consumo de alcohol. ${ }^{(7)}$ Un estudio realizado en España demostró que cerca de la mitad de los estudiantes universitarios refieren su alto nivel de estrés a la cantidad de trabajo y estudio que requieren sus clases y a la falta de tiempo para hacerlo. ${ }^{\left({ }^{8}\right)}$ Se tiende a pensar que la ingestión de alcohol predomina en hombres, sin embargo; hay quienes plantean que la proporción en el consumo de alcohol en mujeres es similar o ligeramente mayor y que esta tendencia podría seguir en aumento. ${ }^{(9)}$

El consumo de etanol es de causa multifactorial; se observa significancia estadística al relacionar las problemáticas mostradas en lo personal, lo sexual y la salud física con el hábito de consumir alcohol.(1) Aún con conocimiento sobre este tema, algunos estudios evidencian solo información y el estudio de contenidos sobre la salud y la enfermedad no son suficientes para que los estudiantes de medicina eviten conductas de riesgo.(10) En Honduras un estudio sobre estilos de vida específicamente en estudiantes de la EUCS/UNAH-VS en el 2015, encontró que el $51 \%$ de la muestra consumía alcohol.(11)

Ya que se cuenta con poca bibliografía nacional referente al consumo de alcohol en estudiantes universitarios de medicina, se pretende que el estudio sirva de base para posteriores investigaciones relacionadas y así contribuir a la formación de políticas y estrategias para la prevención de la ingesta de alcohol. El objetivo principal fue describir 
y comparar entre hombres y mujeres el consumo de alcohol en estudiantes de medicina que cursan Farmacología I y II en el primer semestre del año 2017 en la EUCS/ UNAH-VS.

\section{PACIENTES Y MÉTODOS}

Este fue un estudio cuantitativo con alcance descriptivo realizado, en el primer semestre del año 2017 en estudiantes de Farmacologia I y II de la carrera de medicina de la EUCS/UNAH-VS. Los criterios de inclusión fueron ser estudiante de medicina que curse la asignatura de Farmacología I o II.

La muestra no probabilística estaba compuesta por 56 estudiantes de las asignaturas Farmacología I y II de la carrera de medicina en la EUCS/UNAH-VS. Se trabajó con una muestra por conveniencia de 28 mujeres y 28 hombres.

La recolección de datos se realizó a tráves de un instrumento tipo cuestionario en el cual el estudiante que cumplió con los criterios de inclusión respondió una serie de preguntas cerradas sobre el consumo de alcohol. Los datos se tabularon en Excel, luego se analizaron.

\section{RESULTADOS}

La mayoría de los estudiantes de Farmacología I y II de la EUCS/UNAH-VS encuestados viven en San Pedro Sula y la mayoría consumían alcohol. Destaca en los resultados que son más las mujeres que lo consumen. (Ver tabla No. 1).

Tabla No. 1: Características del consumo de alcohol de los estudiantes de la EUCS/UNAH-VS.

\begin{tabular}{|llcc|}
\hline Variable & Característica & Número & Porcentaje \\
\hline & San Pedro Sula & 36 & $64 \%$ \\
\hline & Otros lugares del país & 20 & $36 \%$ \\
\hline Con quién viven los alumnos & Familia & 46 & $82 \%$ \\
\hline & Vivian solos & 6 & $11 \%$ \\
\hline & Vivian con amigos & 4 & $7 \%$ \\
\hline Consumo de alcohol & $\mathrm{Si}$ & 41 & $73 \%$ \\
\hline & No & 13 & $23 \%$ \\
\hline Consumo de alcohol según sexo & No contestaron & 2 & $4 \%$ \\
\hline & Mujeres que si toman & 24 & $43 \%$ \\
\hline & Mujeres que no toman & 4 & $7 \%$ \\
\hline & Hombres que si toman & 17 & $30 \%$ \\
\hline & Hombresque no toman & 9 & $16 \%$ \\
\hline Consumo de alcohol antes de los 18 años & No contestaron & 2 & $4 \%$ \\
\hline & Mujeres & 15 & $26 \%$ \\
\hline Consumo de alcohol después de los 18 años & Hombres & 12 & $21 \%$ \\
\hline & Mujeres & 9 & $17 \%$ \\
\hline & Hombres & 5 & $9 \%$ \\
\hline & No consumen & 13 & $23 \%$ \\
\hline
\end{tabular}

Fuente: Instrumento sobre consumo de alcohol en estudiantes de Farmacología I y II la EUCS/UNAH-VS.

Las mujeres al ingerir bebidas alcohólicas les gusta comer $38.6 \%$, bailar $36.4 \%$ y practicar juegos de mesa $25 \%$. En los hombres sobresale: comer $41.1 \%$, bailar $26.5 \%$, practican juegos de mesa $20.6 \%$, fumar
$8.8 \%$ y un $3 \%$ refirió no hacer alguna actividad mientras bebe. La bebida preferida por los estudiantes fue la cerveza y el licor. (Ver gráfico No. 1). 
Gráfico No. 1: Preferencia de bebidas de los estudiantes de Farmacología I y II de la EUCS/UNAHVS.

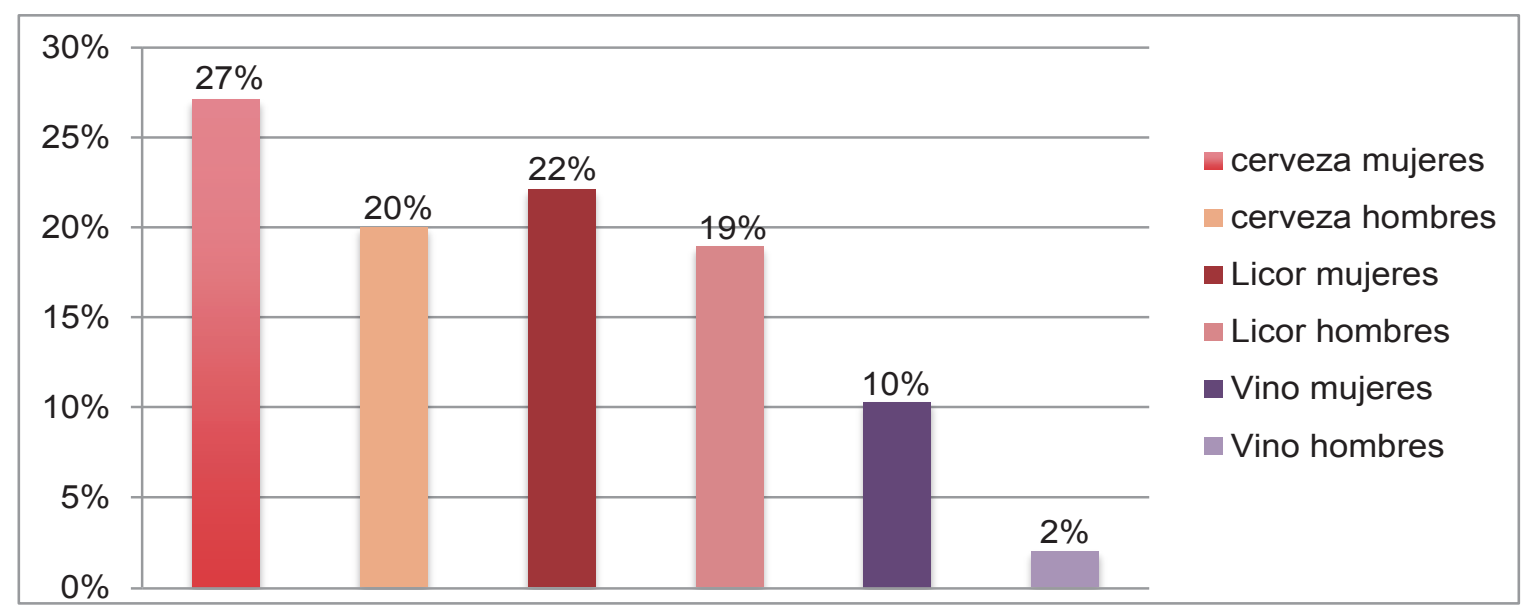

Fuente: Instrumento sobre consumo de alcohol en estudiantes de Farmacología I y II la EUCS/UNAH-VS.

La mujeres prefieren tomar alcohol: en su casa $27 \%$, bar 19.6 , restaurante $5.35 \%$, la playa $5.35 \%$, en cualquier lugar $3.6 \%$ y un $39.1 \%$ no respondió la pregunta. En cuanto a los hombres, los lugares preferidos fueron: su casa $24 \%$, bar $14.3 \%$, paseo $8.9 \%$, alrededor de la universidad $1.8 \%, 49.2 \%$ no tuvo preferencia por el lugar y $1.8 \%$ no respondió a la pregunta. La mayoría prefiere la compañía de amigos para ingerir bebidas alcohólicas y conocen los efectos nocivos del alcohol. (Ver tabla No. 2) sobre el comportamiento que tienen con las bebidas alcoholicas.

Tabla No. 2: Comportamiento con bebidas alcohólicas de los estudiantes de la EUCS/UNAHVS.

\begin{tabular}{|c|c|c|c|}
\hline Variable & Característica & Número & Porcentaje \\
\hline \multirow[t]{5}{*}{ Con quién ingieren bebidas alcohólicas } & Familia & 7 & $18 \%$ \\
\hline & Amigos & 25 & $61 \%$ \\
\hline & Solos & 4 & $9 \%$ \\
\hline & Otros & 1 & $3 \%$ \\
\hline & No respondieron & 4 & $9 \%$ \\
\hline \multirow[t]{5}{*}{ Cuando consumen bebidas alcohólicas } & Vacaciones & 15 & $36 \%$ \\
\hline & Después de examen & 11 & $27 \%$ \\
\hline & Durante todo el período & 2 & $5 \%$ \\
\hline & Al inicio del período & 8 & $20 \%$ \\
\hline & No respondieron & 5 & $12 \%$ \\
\hline \multirow[t]{3}{*}{ Han estado ebrios alguna vez } & (mujeres) & 11 & $27 \%$ \\
\hline & (hombres) & 11 & $27 \%$ \\
\hline & (13 mujeres 6 hombre & es) 19 & $46 \%$ \\
\hline \multirow[t]{5}{*}{ Asisten con resaca a clases } & (mujeres) & 12 & $29 \%$ \\
\hline & (hombres) & 12 & $29 \%$ \\
\hline & (mujeres) & 7 & $17 \%$ \\
\hline & (hombres) & 1 & $4 \%$ \\
\hline & No respondieron & 9 & $21 \%$ \\
\hline \multirow[t]{3}{*}{ Conocen sobre los efectos nocivos del alcohol } & (mujeres) & 24 & $58 \%$ \\
\hline & (hombres) & 16 & $39 \%$ \\
\hline & No respondió & 1 & $3 \%$ \\
\hline \multirow[t]{3}{*}{ Consumo de alcohol } & Moderado (4 bebidas) & 23 & $56 \%$ \\
\hline & (1 bebida ) & 13 & $32 \%$ \\
\hline & No contestarón & 5 & $12 \%$ \\
\hline
\end{tabular}

Fuente: Instrumento sobre consumo de alcohol en estudiantes de Farmacología I y II la EUCS/UNAH-VS. 
El motivo de ingesta alcohólica más frecuente fue por celebración $62.5 \%$ (39.30\% mujeres y $23.20 \%$ hombres). Otros motivos: exámenes aplazados, rompimiento amoroso, influencia de los amigos sumaron un $37.5 \%$. La frecuencia con que consumen alcohol los estudiantes es menos de una vez al mes. (Ver gráfico No. 2).

Gráfico No. 2: Frecuencia de consumo de alcohol distribuido por sexo en los estudiantes de la EUCS/UNAH-VS.

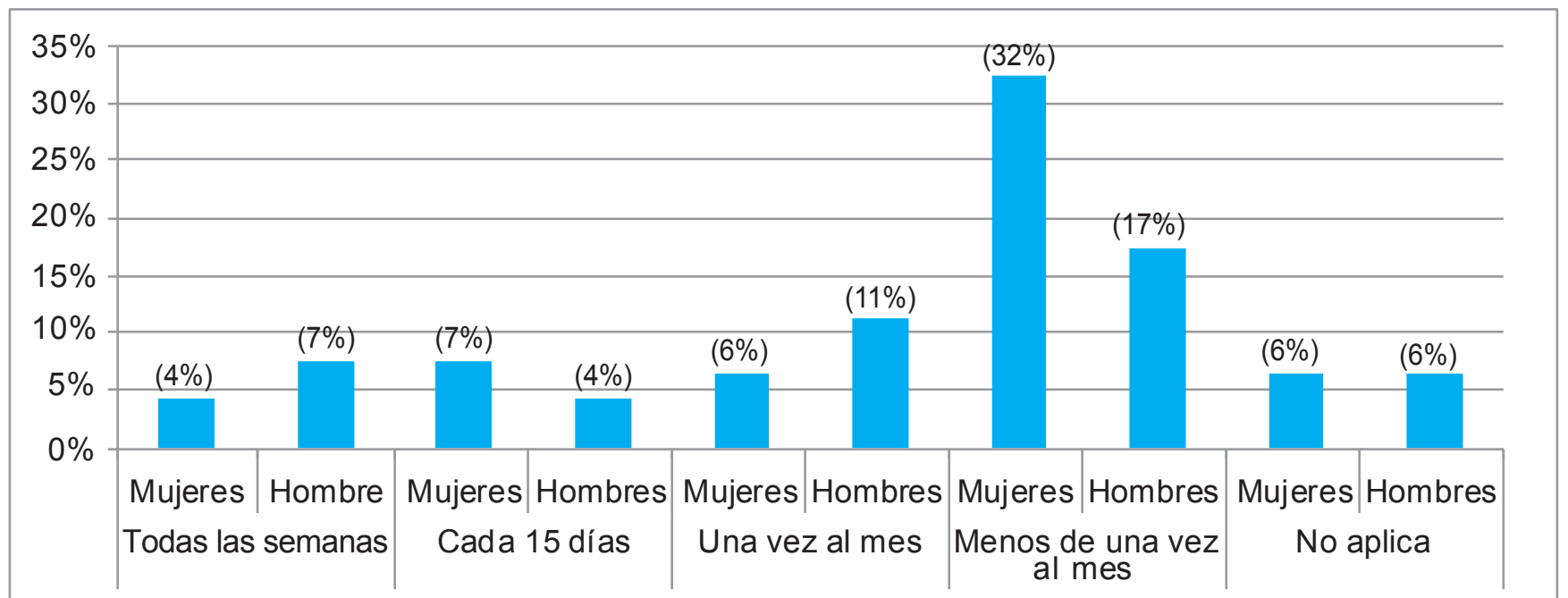

Fuente: Instrumento sobre consumo de alcohol en estudiantes de Farmacología I y II la EUCS/UNAH-VS.

Un poco más del $90 \%$ de los estudiantes refiere que el consumo de alcohol no les afecta sus horas de estudio en ambos sexos.

\section{DISCUSIÓN}

A nivel Iberoamericano, son diversos los estudios sobre el consumo de alcohol en estudiantes universitarios que compartían variables para la comparación de los resultados. Un estudio realizado a universitarios de Medellín, Colombia reflejaron datos de mayor consumo de alcohol en estudiantes mujeres $60.1 \%$ en comparación al $39.9 \%$ en los estudiantes hombres.(1) El mismo comportamiento fue observado en un estudio realizado en la Universidad de Veracruz, México, sobre la prevalencia de consumo de alcohol de estudiantes universitarios, los porcentajes fueron similares con $60.4 \%$ de ingesta de alcohol en estudiantes mujeres y un $39.6 \%$ en estudiantes hombres. ${ }^{(6)}$
El consumo de alcohol en nuestra sociedad suele tener un inicio a edades tempranas de la juventud, esto puede ocasionar un grado mayor de susceptibilidad a enfermedades crónicas u otros padecimientos como alteraciones mentales y enfermedades transmisibles. ${ }^{(3)}$ Este aspecto fué comparado con este estudio en donde se estableció que la mayoría de los casos comenzaron antes de los 18 años, siendo mayor la cantidad de mujeres que la de hombres. En contraste a los porcentajes obtenidos en un estudio realizado en Colombia sobre problemas asociados al consumo de alcohol en estudiantes universitarios, la gran mayoría, $75 \%$ de la muestra aseguró que la edad de inicio en el consumo de este tipo de sustancia fue a partir de los 18 años, ${ }^{(1)}$ otro estudio realizado en México habla de una edad promedio de inicio de consumo de alcohol a los 16 años. ${ }^{(6)}$

Otra de las investigaciones a considerar, al igual que en nuestro estudio, es el tipo de 
bebida de preferencia dentro de la población estudiantil en la cual se presentaba opciones como cerveza, licores, vino, etc. donde prevaleció la cerveza con un $53 \%,{ }^{(6)}$ similar al porcentaje obtenido en esta investigación $44.64 \%$. Notable destacar que en la mayoría de los casos se consumían combinaciones de diferentes tipos de bebidas antes mencionados.

El consumo de alcohol es poco frecuente, sin embargo la cantidad es moderada en los estudiantes. En un estudio sobre la familia y consumo de alcohol en universitarios reporta datos de un consumo sensato a moderado de estas sustancias $45.2 \%$, y en menor grado un consumo perjudicial $26.2 \%,{ }^{(8)}$ similar a nuestro estudio en el que un $41.07 \%$ tenía un consumo moderado, pero contrasta en el consumo perjudicial, ya que solo el $9.52 \%$ lo tenía. Otra investigación estipuló la frecuencia en la que los estudiantes consumen alcohol, un $20 \%$ aseveró hacerlo 1 a 3 veces por mes, ${ }^{(6)}$ en contraste con los resultados expuestos aquí donde solo el $11 \%$ (7\% mujeres y $4 \%$ hombres) consumía alcohol cada 15 días.

Datos en Colombia revelaron que muchos acompañaban sus bebidas con el cigarriIlo, ${ }^{(12)}$ a diferencia de ésta investigación en donde solo un pequeño porcentaje acompañaba sus bebidas con cigarrillos, $8.82 \%$ en su totalidad hombres.

En cuanto a la relación con el rendimiento académico, estudios establecen que un $87.7 \%$ de los estudiantes aseguran no tener problemas con sus asistencias a clase a raíz del consumo de alcohol, ${ }^{(1)}$ similar a los resultados obtenidos en los que se demostró que los estudiantes no presentaban interrupciones con sus horas de estudio por el consumo de alcohol.

Otro estudio realizado en España sobre consumo de alcohol en la adolescencia: consideraciones médicas y orientaciones educati- vas, reconocen que guiados principalmente por el placer momentáneo asociado a la bebida, forzados por la presión del grupo de amigos, impulsados por sus deseos de mejorar la comunicación con los demás o como forma de evasión, los humanos obvian en gran medida los daños asociados al consumo. ${ }^{(13)}$ Los resultados de ésta investigación concuerdan en que casi la totalidad (98\%) siguen consumiendo a pesar de conocer las consecuencias perjudiciales.

\section{Conclusión}

El consumo de alcohol es considerado un problema grave a nivel mundial. Los estudiantes de Farmacología I y II de la EUCS/ UNAH-VS son un grupo poblacional que, en su mayoría mujeres, ha experimentado el consumo de alcohol al menos una vez o lo hacen ocasionalmente y no interfiere con las actividades curriculares. La sustancia alcohólica más consumida es la cerveza y el licor por su bajo costo y mayor acceso. Los jóvenes universitarios prefieren la comodidad de su casa para consumir las bebidas alcohólicas y usualmente las acompañan con bocadillos, juegos y bailes. La resaca no evita que asistan a clases, sin embargo, la mayoría prefiere beber una vez finalizado sus exámenes o en período de vacaciones. La mayoría de los estudiantes conocen los efectos nocivos del alcohol pero eso no los detiene a consumirlo.

\section{Recomendaciones}

Recomendamos a futuros investigadores a seguir indagando sobre el consumo de alcohol en estudiantes de medicina, a los docentes a impartir talleres enfocados a las consecuencias del alcohol en el organismo y a las autoridades de la universidad a brindar espacios para practicar deportes disponibles hasta los fines de semana, talleres gratuitos sobre temas que les servirán a los estudiantes de medicina en el futuro. Todo esto para educar y evitar que nuestra población joven se vea envuelta en vicios que limitan sus capacidades como estudiantes. 


\section{REFERENCIAS BIBLIOGRÁFICAS}

1. Castaño-Pérez GA, Calderón Vallejo GA. Problemas asociados al consumo de alcohol en estudiantes universitarios. Rev Latino-Am Enfermagem. 2014; 22(5): 739-46.

2. Romero LD, Rojas DA, Zamora A. Consumo de alcohol en los estudiantes de la facultad de salud de la universidad sur colombiana. RFS. 2013;5(1):65-71.

3. Organización Mundial de la Salud. Glosario de términos de alcohol y drogas [Internet]. Washington: OMS; 1994 [Consultado 24 Mayo, 2017]. Disponible en: http://www.who.int/substance_abu se/ter minology/who_ladt/en/.

4. Borrás Santiesteban T, Borrás AR. EI tema "Consumo de bebidas alcohólicas en la adolescencia" en la formación de profesionales de la salud en Cuba. CCM. 2016; 20(2):372-82.

5. Rodríguez-Torres E, Camargo-Restrepo C, Cardona-Blandón AM, Lozano-Sandoval NX, López-Medina DI, Rosas-Villamizar NI. Consumo de alcohol y otras sustancias, un problema en estudiantes de universitarios de salud. Rev Cienc Cuidad. 2016;13(1):59-71.

6. Barradas Alarcón M, Fernández Mojica N, Gutiérrez Serrano L. Prevalencia de consumo de alcohol en estudiantes universitarios. RIDE. 2016;6(12).

7. Hinojosa García L, Gherardi-Donato EC, Alonso-Castillo MM, Cocenas da Silva R, García García P, Maldonado-García V.
Estrés académico y consumo de alcohol en universitarios de nuevo ingreso. Journal Health NPEPS. 2017;2(1):133-147.

8. Armendáriz García NA, Alonso Castillo MM, Alonso Castillo BA, López Cisneros MA, Rodríguez Puente LA, Méndez Ruiz MD. La familia y el consumo del alcohol en estudiantes universitarios. Cienc Enferm. 2014;20(3):109-18.

9. Campo Y, Pombo LM, Teherán A. Estilos de vida saludable y conductas de riesgo en estudiantes de medicina. Rev Univ Ind Santander. 2016;48(3):301-309.

10. Gárciga Ortega $O$, Surí Torres $C$, Rodríguez JR. Consumo de drogas legales y estilo de vida en estudiantes de medicina. Rev Cubana Salud Pública. 2015; 41(1): 4-17.

11. Miranda Baquedano VM, Ayes Panchamé DM. Estilos de vida que practican estudiantes de la Escuela Universitaria de Ciencias de la Salud. Rev Cienc Esc Univ Cienc Salud. 2015;2(2):30-39.

12. Mantilla-Toloza SC, Villamizar CE, Peltze K. Consumo de alcohol, tabaquismo y características sociodemográficas en estudiantes universitarios. Rev Univ salud. 2016;18(1):7-15.

13. Rodríguez García FD, Sanchiz Ruiz ML, Bisquerra Alzina R. Consumo de alcohol en la adolescencia. Consideraciones médicas y orientaciones educativas. Salud Mental. 2014;37(3): 255-60. 